\title{
Amsterdaml'og
}

\section{Title: Preconditioning Techniques for Unconventional Well Testing Problems}

Michel A. Cancelliere (Politecnico di Torino)

\begin{abstract}
Nowadays the environmental issue is of primary interest in well-testing, since the conventional production-build-up sequence for reservoir appraisal are difficulty accepted by some government. Attention is now focused on long injection/fall-off tests that eliminate surface emissions and can significantly reduce testing costs. This approach, however, increase difficulties in the well-test interpretation because of the presence of two mobile phases in the reservoir: the fluid originally in place (hydrocarbon) and the injected fluid (typically diesel or brine). In such systems, saturation profiles are governed by effective permeabilities characterized by variation during the injection phase. Only very favourable mobility ratios show displacement that can be described by a piston like model. Moreover gravitational and thermal gradients, heterogeneity and anisotropy might strongly affect the variation on fluid distribution with time, resulting in an inadequacy of analytical models to describe the pressure transient behaviour. Only numerical simulation can thoroughly describe the saturation and pressure field in the reservoir as it evolves during injection and subsequent fall-off.
\end{abstract}

Verga et. al. ${ }^{l}$ has developed and implemented a 3D axial-symmetric mode which takes into account all the aspects that can impact on fluid and pressure distribution. The sparsity and the ill-conditioning of the derived system suggested a performance analysis of the numerical simulator.

In the resolution of the non-linear algebraic system resulting from the finite differences discretization of the considered 3D axial-symmetric model, using the family of Newton-type methods, is necessary to deal with large sparse and ill conditioned matrices. That is why iterative methods based on Krylov subspaces are usually suitable for this type of problem. However, to improve the convergence of such methods is essential to use preconditioning methods. There are several preconditioning techniques available in the literature are characterized by a lack of robustness, that is they fit to a specific problems. Therefore to achieve optimum results, it is necessary to use preconditioners suitable to each specific type of problem.

The main objective of the work was the analysis of the most appropriated preconditioning technique for Unconventional Well Testing numerical simulations.

The specific objective was an efficient implementation of a proconditioner as a sub-block of a simulator already implemented. The deliverable is a plug-in capable of taking the linear system, represented by the Jacobian of the equation of Newton, solving it by a preconditioned

\footnotetext{
${ }^{1}$ Verga, F., Viberti, D., Salina Borello, E. A new numerical method to interpret injection Tests. Proc 8th Offshore Mediterranean Conference and Exhibition, Ravenna, Italy, 28-30 March.
} 


\section{Amsterdaml'og}

iterative method (GMRES) and returning the result, looking for a good compromise between efficiency and robustness.

The work has been subdivided into two parts: the former consisted in a theoretical study of the preconditioners available and the mathematical structure of the Jacobian, while the latter was focused on the search of a computationally efficient way to implement the previously selected preconditioners.

Under this premise the library PETSc (Portable, Extensible Toolkit for Scientific Computation) has been identified as an essential tool to perform the task. This library actually is capable of handling large systems in an efficient way, and offers the possibility to link with external programs. The use of the library PETSc also opens the way to parallel implementation of the code since it is based on MPI (Message Passing Interface) architecture.

For the study of the various preconditioners a sensitivity analysis of the following parameters has been done: relative permeabilities, perforated interval, problem dimension, grid, time step.

The previous parameters were tested for each preconditioners and the resulting efficiency has been studied.

There has been a large increase in the efficiency of the system when using the right preconditioner, depending on the system studied. It is noted that the classic techniques do not always work well. Moreover it is observed that preconditioners currently used in Reservoir Simulation may not be as efficient in Well Testing problems.

The knowledge of the best technique of preconditioning for unconventional well testing problems can significantly improve the performance of the simulation, reducing the computation time and increasing the overall efficiency of the simulation process.

Several studies of preconditioners have been conducted over the years in the reservoir simulation field, however, analysis of Well Testing specific problem are hard to find. That is why this work may be used as a reference point in the selection of preconditioning techniques for this type of problem. 